\title{
An Optimal TDMA-Based MAC Scheduling for the Minimum Energy Multicast in Wireless Ad Hoc Networks
}

\author{
Song Guo and Oliver Yang \\ School of Information Technology and Engineering \\ University of Ottawa, Ottawa, Ontario, Canada \\ sguo@site.uottawa.ca
}

\begin{abstract}
Energy conservation is a critical issue in wireless ad hoc networks since batteries are the only limitedlife energy source to power the nodes. The introduction of real-time applications has posed additional challenges. Transmission of video and imaging data requires both energy and QoS aware routing in order to ensure efficient usage of the networks. In this paper, we only consider "bandwidth" as the QoS in TDMA-based wireless ad hoc networks that use omni-directional antennas and have limited energy resources. We present a constraint formulation model for the QoS-MEM (QoS-aware Minimum Energy Multicast) problem in terms of mixed integer linear programming (MILP). It can be used for an optimal solution of the QoS-MEM problem, and it also provides a way to evaluate the realistic performance of different heuristic algorithms.
\end{abstract}

\section{Introduction}

The emergence of real-time applications and the widespread use of wireless devices have generated the need to provide quality-of-service (QoS) support in wireless ad hoc networking environments. Since wireless nodes are generally dependent on finite battery source, the routing protocol for QoS provisioning must also consider the residual battery power and the rate of battery consumption in order to increase longevity of such networks $[1,2]$. Thus all the techniques for QoS provisioning should be powerefficient. On the other hand, the ability to provide QoS is heavily dependent on how well the resources are managed at the MAC layer. A QoS routing protocol developed for one type of MAC layer does not generalize to others easily. Among the QoS routing protocols proposed so far, some use generic QoS measures and are not tuned to a particular MAC layer [3-5]. Some use CDMA to eliminate the interference between different transmissions [6, 7]. In [8], the authors develop a QoS routing protocol for ad hoc networks using TDMA in small networks.

The recent proliferation of QoS-aware group applications over the wireless ad hoc networks has accelerated the need for efficient multicast support. In this paper, we only consider "bandwidth" as the QoS and present an integer linear programming (MILP) formulation model for the QoS-MEM (QoS-aware Minimum Energy Multicast) problem in a TDMAbased ad hoc network. Clearly, such a joint powerminimization and scheduling is a challenging optimization problem. In fact, either the scheduling problem with even a single power level or the besteffort minimum energy multicast problem, is by itself known to be an NP-hard problem proven in $[9,10]$ respectively. Our MILP model can be used for an optimal solution of the QoS-MEM problem, and thus provides a way to evaluate the realistic performance of different heuristic algorithms.

\section{Network Model}

Let us model the wireless ad hoc network by a simple directed graph $G(N, A)$, where $N$ is a finite node set, $|N|=n$, and $A$ is an arc set corresponding to the unidirectional wireless communication links. Each node is equipped with a single omni-directional antenna. When considering uniform propagation condition, we observe that all nodes within the communication range of a transmitting node can receive its transmission, and the received signal power varies as $r^{-\alpha}$, where $r$ is the distance to the sender, and $\alpha$ is propagation loss exponent that typically takes on a value between 2 and 4 , depending on the characteristics of the communication medium. We further assume that any node $v \in N$ can choose its transmission power level $p_{v u}$ continuously up to some maximum value $p_{v}{ }^{\max }$.

In this paper we shall develop a constraint formulation for the QoS-MEM problem in ad hoc networks using TDMA, in which all the nodes are 
synchronized. We assume that any node can only receive a single transmission at a time and cannot transmit and receive simultaneously. The bandwidth is partitioned into a set of time slots $S=\{1,2, \ldots, K\}$ which consist the data part of a frame. The information concerning available bandwidth (in number of free time slots) between two nodes is critical, because it is used to select a route that satisfies the QoS requirement. In addition, it is also used to determine whether a new connection request is allowed into the network.

Let $P_{u i}(u \in N)$ be the power level in the slot $i$ assigned to node $u$, where $0 \leq P_{u i} \leq p_{u}{ }^{\max }$ and $1 \leq i \leq K$. The transmission schedule $T S_{u}$ of node $u \in N$ is thus defined as the power assignment in each time slot, i.e. $T S_{u}=\left(P_{u 1}, P_{u 2}, \ldots, P_{u K}\right)$. For any new traffic request, based on its current transmission schedule $T S_{u}$, a transmission from node $u$ can be only scheduled in a set of free time slots $F S_{u}$, define as $F S_{u}=\left\{i \mid P_{u i}=0, i\right.$ $\in S\}$. Note that $F S_{u}$ is only an alternate set for scheduling, and may not guarantee conflict-free transmission. We say a transmission from node $v$ is successfully received at node $u$ in the slot $i$ if and only if the signal-to-interference plus noise ratio (SINR) at $u$ is not less than the minimum required threshold $\gamma$, i.e.

$$
\frac{P_{v i} / r_{v u}^{\alpha}}{\eta+\sum_{(x, u) \in A, x \neq v}\left(P_{x i} / r_{x u}^{\alpha}\right)} \geq \gamma
$$

where $r_{v u}$ is the distance between nodes $v$ and $u$, and $\eta$ is the thermal noise at every receiver.

\section{Constraints Formulation}

We consider a source-initiated multicast in wireless ad-hoc networks. Any node is permitted to initiate multicast sessions. Multicast requests and session durations are generated randomly at the network nodes. A multicast session involves a set of multicast nodes $M$ (including the source node and all destination nodes) and non-member nodes that relay traffic to support the multicast communication. Multicast employs a tree structure in the network to efficiently deliver the same data stream to a group of receivers. Formally, we define $T_{s}\left(N^{\prime}, A^{\prime}\right)$ to be a bandwidth-constrained multicast tree (BCMT) of $G(N, A)$ rooted at $s$ with a multicast node set $N^{\prime} \subseteq N$, and an arc set $A^{\prime} \subseteq A$, if and only if the following constrains are satisfied. 1) RTC (Rooted Tree Constraint): this constraint requires $T_{s}$ to be a rooted tree and span all the multicast members from node $s$, i.e. $M \subseteq N^{\prime} ; 2$ ) BWC (Bandwidth Constraint): this constraint requires that the bandwidth allocated on each link of the multicast tree should meet the bandwidth requirement ( $B$ slots per frame), and the scheduling should be conflict-free.
The definition of bandwidth-constrained multicast tree allows us to formulate the QoS-MEM Problem as a MILP (Mixed Integer Linear Programming) model. The main idea is to extract a sub-graph $T_{s}{ }^{*}$ from the original graph $G$, such that $T_{s}{ }^{*}$ is a BCMT with minimum energy consumption. In order to formulate the problem, we define the following decision variables: i) $Z_{v u}$ is a binary variable which is equal to one if the $\operatorname{arc}(v, u)$ is in the sub-graph $T_{s}{ }^{*}$ of $G$, and zero otherwise; ii) $F_{v u}$ is a nonnegative continuous variable that only represents fictitious flow produced by the multicast initiator $s$ going through arc $(v, u)$, and thus helps prevent loops; iii) $\quad q_{u i}$ is a nonnegative continuous variable which represents the transmission power of the node $u$ in slot $i$; and iv) $t_{v u i}$ is a binary variable which is equal to one if node $v$ is scheduled to transmit to node $u$ in slot $i$, and zero otherwise.

Let $T S_{u}=\left(P_{u 1}, P_{u 2}, \ldots, P_{u K}\right), u \in N$, be the current conflict-free transmission schedule (before the multicast request). We note that if there are certain time slots already reserved in the network, for example the slot $i$ reserved for transmission from node $v$ to $u$ with transmission power $P_{v i}$, the values of the decision variables $q_{v i}$ and $t_{v u i}$ should be preset as $q_{v i}=P_{v i}$ and $t_{v u i}$ $=1$. For those unscheduled slots $\left(P_{v i}=0\right)$, the values of variables $q_{v i}$ and $t_{v u i}$ would be obtained after the optimization problem is solved.

We shall prove that if $(x)^{*}$ is the optimal solution of variable $x$ obtained from this MILP model, then the graph $T_{s}^{*}\left(N^{\prime}, A^{\prime}\right)$ is the optimal tree associated with this solution, i.e. $T_{s}^{*}\left(N^{\prime}, A^{\prime}\right)$ is a BCMT of $G$ with minimum energy consumption. In this graph, $N^{\prime}=\{u \mid \exists(v, u) \in$ $A^{\prime}$ or $\left.(u, v) \in A^{\prime}\right\}$ is its arc set, and $A^{\prime}=\left\{(v, u) \mid Z_{v u}^{*}=\right.$ $1\}$ is its arc set. In the following, we formulate all the constraints for the Problem QoS-MEM.

In the following, we want to provide a set of constraints that would guarantee that $T_{s}{ }^{*}\left(N^{\prime}, A^{\prime}\right)$ obtained from the formulation satisfies the rooted tree property. It can be characterized that $T_{s}{ }^{*}\left(N^{\prime}, A^{\prime}\right)$ is a rooted tree spanning all the multicast members, i.e., $M$ $\subseteq N^{\prime}$, by the following constraints. a) Every node $u, u$ $\in N^{\prime}-\{s\}$, has exactly one incoming arc, and node $s$ has no incoming arcs. b) $T_{s}^{*}\left(N^{\prime}, A^{\prime}\right)$ does not contain cycles. The construction of the linear constraints for these two properties are elaborated in the following theorems.

Theorem 1. $T_{s}{ }^{*}\left(N^{\prime}, A^{\prime}\right)$ is a directed graph in which node $s$ has no incoming arcs, and each other node has exactly one incoming arc, provided Problem QoSMEM satisfies the following constraints:

$$
\sum_{v:(v, u) \in A} Z_{v s}=0
$$




$$
\begin{array}{rlrl}
\sum_{v:(v, u) \in A} Z_{v u} & =1 ; & \forall u \in M-\{s\} \\
\sum_{v:(v, u) \in A} Z_{v u} \leq 1 ; & \forall u \in N-M \\
\sum_{v:(u, v) \in A} Z_{u v} \leq(n-1) \sum_{v:(v, u) \in A} Z_{v u} ; & \forall u \in N-M
\end{array}
$$

Theorem 2. $T_{s}{ }^{*}\left(N^{\prime}, A^{\prime}\right)$ does not contain cycles, if Problem QoS-MEM satisfies the constraints (2) to (4) and the following constraints:

$$
\begin{aligned}
& \sum_{v:(v, u) \in A} F_{v u}-\sum_{v:(u, v) \in A} F_{u v}=\sum_{v:(v, u) \in A} Z_{v u} ; \quad \forall u \in N-\{s\} \\
& Z_{v u} \leq F_{v u} \leq(n-1) Z_{v u} ; \quad \forall u \in N-\{s\},(v, u) \in A
\end{aligned}
$$

The bandwidth constraints reflect the conditions that bandwidth allocated on each link of the multicast tree should be conflict-free and meet the bandwidth requirement, which can be characterized as follows. a) Along each wireless link $(v, u)$ in the optimal multicast tree $T_{s}{ }^{*}\left(Z_{v u}^{*}=1\right)$, the transmissions for this multicast session are scheduled in a set of free time slots $\left\{i \mid t^{*}{ }_{\text {vui }}\right.$ $\left.=1, i \in F S_{v}\right\}$ with cardinality of $B$ (the bandwidth requirement), i.e. $\sum_{i \in F S_{v}} t_{v u i}^{*}=B$. For non-multicast link $\left(Z_{v u}^{*}=0\right)$, no additional bandwidth should be reserved, i.e. $\sum_{i \in F S_{v}} t_{v u i}^{*}=0$. b) If the current transmission schedule (before the multicast request) $T S_{u}=\left(P_{u 1}, P_{u 2}, \ldots, P_{u K}\right)$ is conflict-free, then the transmission schedule $T S_{u}^{\prime}{ }_{u}=$ $\left(q^{*}{ }_{u 1}, q_{u 2}^{*}, \ldots, q_{u K}^{*}\right)$ is also conflict-free after the multicast with required bandwidth is allowed into the network. The following theorem explains how the BWC (a) can be achieved by a set of linear constraints.

Theorem 3. $T_{s}{ }^{*}\left(N^{\prime}, A^{\prime}\right)$ satisfies the bandwidth requirement, if the formulation of Problem QoS-MEM includes the constraints (8) and (9).

$$
\begin{aligned}
& t_{v u i} \leq Z_{v u} ; \quad \forall(v, u) \in A, \forall i \in F S_{v} \\
& \sum_{v \cdot(v, u) \in A} \sum_{i \in F S} t_{v u i}=B \cdot \sum_{(v, u) \in A} Z_{v u} ; \quad \forall u \in N
\end{aligned}
$$

The last set of constraints we need to build up is the conflict-free condition, which requires that at any time slot and any receiving node, the SINR requirement (Equation 1) should be satisfied. That is, the new reservation for the multicast session would not result in any conflict either with reservations established earlier or within the multicast traffic itself that is allowed to enter into the network.

Theorem 4. The new transmission schedule $\left(q^{*}{ }_{u 1}\right.$, $\left.q^{*}{ }_{u 2}, \ldots, q_{u K}^{*}\right)$ is conflict-free, if the formulation of Problem QoS-MEM includes Constraints (10) to (12), where $\beta$ is a relatively large number.

$$
\sum_{v:(u, v) \in A} t_{u v i} \leq(n-1)\left(1-\sum_{v:(v, u) \in A} t_{v u i}\right) ; \quad \forall i \in S, \forall u \in N
$$

$$
\begin{aligned}
& \frac{q_{v i}}{r_{v u}^{\alpha}}-\gamma\left(\eta+\sum_{\substack{x:(x, u) \in A \\
x \neq v}} \frac{q_{x i}}{r_{x u}^{\alpha}}\right) \geq \beta\left(t_{v u i}-1\right) ; \forall i \in S, \forall(v, u) \in A \\
& 0 \leq q_{u i} \leq p_{u}^{\max } ; \\
& \forall u \in N
\end{aligned}
$$

\section{Conclusion}

In this paper we present a constraint formulation for the bandwidth-guaranteed minimum-energy multicast problem in multi-hop ad hoc wireless networks. Based on the analysis on the properties of multicast tree and conflict-free scheduling, the problem can be characterized in a form of mixed integer linear programming problem. To our best knowledge, this is the first work using mixed integer linear programming to formulate this problem. Many application scenarios can be solved efficiently based on the formulation using branch-and-cut or cutting planes techniques. The optimal solutions can be used to assess the performance of heuristic algorithms for mobile networks by running them at discrete time instances.

\section{References}

[1] C. Diot, W. Dabbous, and J. Crowcroft, "Multipoint communication: a survey of protocols, functions, and mechanisms," IEEE Journal on Selected Areas in Communications, 15, pp. 277-290, April 1997.

[2] M. H. Ammar, G. C. Polyzos, and S. K. Tripathi, "Special issue on network support for multipoint communication," IEEE Journal on Selected Areas in Communications, 15, April 1997.

[3] S. Lee and A. T. Campbell, "INSIGNIA: In-band signalling support for QoS in mobile ad hoc networks", in Proceedings of the 5th International Workshop on Mobile Multimedia Communication, 1998.

[4] S. Chen and K. Nahrstedt, "Distributed Quality-ofService in Ad Hoc Networks", IEEE Journal on Selected Areas in Communications, 17(8), 1999.

[5] E. M. Royer, C. Perkins, and S. R. Das, "Quality of Service for Ad Hoc On-Demand Distance Vector Routing", in Internet-Draft, draftietf-manet-aodvqos00.txt, July 2000

[6] C. R. Lin and J. S. Liu, "QoS Routing in Ad Hoc Wireless Networks", IEEE Journal on Selected Areas in Communications, 17(8), pp.1426-1438, 1999.

[7] C. R. Lin, "On-demand QoS routing in multihop mobile networks", in Proceedings of IEEE INFOCOM, 2001.

[8] Chenxi Zhu and M. Scott Corson, "QoS routing for mobile ad hoc networks", in Proceedings of IEEE INFOCOM, 2002.

[9] E. Arikan, "Some complexity results about packet radio networks", IEEE Transaction on Information Theory, July 1984.

[10] Mario Cagalj, Jean-Pierre Hubaux, and Christian Enz, "Minimum-energy broadcast in all-wireless networks: NP-completeness and distribution issues", Proceedings of ACM MOBICOM, Atlanta, Georgia, USA, 2002, pp. 172-182. 